\title{
Trapping constant, thermal conductivity, and the microstructure of suspensions of oriented spheroids
}

\author{
S. Torquato ${ }^{a)}$ \\ Courant Institute of Mathematical Sciences, New York University, 251 Mercer Street, \\ New York, New York 10012 \\ F. Lado \\ Department of Physics, North Carolina State University, Raleigh, North Carolina 27695-8202
}

(Received 10 October 1990; accepted 6 December 1990)

\begin{abstract}
The $n$-point probability function $S_{n}\left(\mathbf{r}^{n}\right)$ is fundamental to the study of the macroscopic properties of two-phase random heterogeneous media. This quantity gives the probability of finding $n$ points with positions $\mathbf{r}^{n} \equiv\left\{\mathbf{r}_{1}, \ldots, \mathbf{r}_{n}\right\}$ all in one of the phases, say phase 1 . For media composed of distributions of oriented, possibly overlapping, spheriods of one material with aspect ratio $\epsilon$ in a "matrix" of another material, it is shown that there is a scaling relation that maps results for the $S_{n}$ for sphere systems $(\epsilon=1)$ into equivalent results for spheriod systems with arbitrary aspect ratio $\epsilon$. Using this scaling relation it is then demonstrated that certain transport and microstructural properties of spheriodal systems generally depend upon purely shape-dependent functions and lower-order spatial moments of $S_{2}$ (minus its long-range value) of the equivalent spherical system. Specifically, the following three distinct calculations are carried out for both hard, oriented spheroids and overlapping (i.e., spatially uncorrelated), oriented spheroids: (1) bounds on the diffusion-controlled trapping constant; (2) bounds on the effective conductivity tensor; and ( 3 ) fluctuations in the local volume fraction as measured by the "coarseness." These computations enable us to investigate the effects of statistical anisotropy (i.e., particle asymmetry) and particle exclusion volume on the aforementioned quantities.
\end{abstract}

\section{INTRODUCTION}

The study of the macroscopic properties (e.g., transport, mechanical, optical, and electromagnetic properties) and microstructure of two-phase heterogeneous media has a long history and has been receiving considerable attention in the last decade (see Refs. 1-3 and references therein). Many natural and artificial materials are heterogeneous on a "microscopic" scale, which is much smaller than the macroscopic length scale but much larger than molecular length scales. This class of materials include fluid-saturated sandstones, blood, animal and plant tissue, slurries, microemulsions, laminated composites, fiber-reinforced materials, particulate composites, cermets, and soils, to mention but a few sxamples.

The effective properties of such media generally depend upon the microstructure via an infinite set of statistical correlation functions. This set of correlation functions is not unique, however. ${ }^{4}$ One such correlation function is the $n$ point probability function $S_{n}\left(\mathbf{r}^{n}\right)$, which gives the joint probability of finding $n$ points with positions $\mathbf{r}^{n}$ $\equiv\left\{\mathbf{r}_{1}, \mathbf{r}_{2}, \ldots, \mathbf{r}_{n}\right\}$ all in one of the phases, say phase 1 . Rigorous expressions for the effective conductivity of composites, ${ }^{2,3,5-7}$ trapping constant associated with diffusioncontrolled reactions among static traps ${ }^{8}$ fluid permeability associated with viscous flow through porous media, ${ }^{9,10}$ and the elastic moduli of composites, ${ }^{11}$ have been shown to depend upon functionals of the $S_{n}$.

\footnotetext{
"On leave of absence from the Department of Mechanical and Aerospace Engineering, North Carolina State University, Raleigh, NC 27695-7910.
}

In recent years progress has been made in representing and computing the $S_{n}$ for isotropic two-phase random-media models consisting of distributions of spheres of one material in a "matrix" of another material., ${ }^{72-14}$ This has led to the evaluation of rigorous bounds on the effective properties of such models. ${ }^{3,6,-10,15,16}$ Only a few calculations of lower-or$\operatorname{der} S_{n}$ for statistically anisotropic distributions of particles have been carried out to date. The two-point function $S_{2}$, for example, has only recently been computed for overlapping (i.c., spatially uncorrclated), oricnted cylinders of finite aspect ratio $^{7}$ and for oriented, nonoverlapping (i.e., spatially correlated) spheroids of arbitrary aspect ratio. ${ }^{17}$ Consequently, the problem of rigorously determining the macroscopic properties of statistically anisotropic two-phase random media has been relatively unexplored.

One of the purposes of this paper is to further study the microstructure of distributions of oriented, possibly overlapping, spheroids of aspect ratio $\epsilon=b / a$. The axially symmetric sphcroidal inclusions, with semiaxes $a$ and $b$, are aligned with their symmetry axis in the $z$ direction. This is a useful model of statistically anisotropic two-phase media, containing the special limiting cases of oriented disks $(\epsilon=0)$, spheres $(\epsilon=1)$ and oriented needles $(\epsilon=\infty)$ and may be generally employed to model anisotropic sandstones, laminates, and aligned, short-fiber composites.

We show in Sec. II that there is a scaling relation that enables one to map results for the $S_{n}$ for sphere systems $(\epsilon=1)$ into equivalent results for spheroids with arbitrary aspect ratio $\epsilon$. Using this scaling relation we then demonstrate in Secs. III-V that certain transport and microstructural properties of spheroidal systems generally depend upon 
purely shape-dependent functions and lower-order spatial moments of the two-point function $S_{2}$ (minus its long-range value $S_{1}^{2}$ ) of the equivalent spherical system.

In particular, we consider the following three distinct calculations for both hard (i.e., spatially correlated) and overlapping (i.e., spatially uncorrelated) oriented spheroids:

(1) two-point bounds on the diffusion-controlled trapping constant ${ }^{8} \gamma$;

(2) two-point bounds on the effective conductivity ten$\operatorname{sor}^{7} \boldsymbol{\sigma}_{e} ;$ and

(3) the so-called coarseness ${ }^{16} C$ (i.e., local volume fraction fluctuations), a meausre of the nonuniformity of spatial coverage of the inclusions.

These computations will enable us to study the effects of statistical anisotropy (by varying $\epsilon$ ) and particle exclusion volume on the aforementioned quantities.

\section{MICROSTRUCTURE CHARACTERIZATION}

Consider statistically inhomogeneous media composed of identical, oriented inclusions of arbitrary shape (phase 2) with volume fraction $\phi_{2}$ distributed throughout a "matrix" (phase 1) with volume fraction $\phi_{1}$. The matrix space need not be continuously connected since the inclusions are generally allowed to overlap one another, creating a possibly connected "particle" phase. Torquato and Stell 12,13 were the first to consider obtaining the $S_{n}\left(\mathbf{r}^{n}\right)$ for statistically inhomogeneous distributions of identical spheres in terms of the $n$-particle probability density functions $\rho_{1}, \ldots, \rho_{n}$. The quantity $\rho_{n}\left(\mathbf{r}^{n}\right)$ characterizes the probability of finding a configuration of $n$ spheres with positions $\mathbf{r}^{n}$. Thus, $S_{n}\left(r^{n}\right)$ gives the probability of finding $n$ points with positions $r^{n}$ in the matrix phase. Torquato and $\mathrm{Sen}^{7}$ generalized the Torquato-Stell sphere results to cases of identical inclusions in which the configurational coordinate for each inclusion is fully specified by its center-of-mass position (e.g., oriented cylinders or ellipsoids) by a simple reinterpretation of the inclusion indicator function; they found

$$
\begin{aligned}
S_{n}\left(\mathbf{r}^{n}\right)= & 1+\sum_{k=1}^{\infty} \frac{(-1)^{k}}{k !} \int \rho_{k}\left(\mathbf{r}_{n+1}, \ldots, \mathbf{r}_{n+k}\right) \\
& \times \prod_{j=n+1}^{n+k}\left(1-\prod_{i=1}^{n}\left[1-m\left(\mathbf{r}_{j i}\right)\right]\right) d \mathbf{r}_{j},
\end{aligned}
$$

where

$$
m(\mathbf{r})= \begin{cases}1, & \mathbf{r} \in D_{l}, \\ 0, & \text { otherwise }\end{cases}
$$

is the inclusion indicator function, $D_{I}$ the inclusion region, $\mathbf{r}$ a position vector measured with respect to the inclusion centroid, and $\mathbf{r}_{j i}=\mathbf{r}_{i}-\mathbf{r}_{j}$. Note that in relation (2.1) the positions $\mathbf{r}_{1}, \ldots, \mathbf{r}_{n}$ refer to points in the matrix while the positions $\mathbf{r}_{n+1}, \ldots, \mathbf{r}_{n+k}$ refer to the inclusion centroids. For the special case of axially symmetric spheroids with semiaxes $a$ and $b$ aligned with their symmetry axis in the $z$ direction, one has

$$
m(\mathbf{r})= \begin{cases}1, & \left(x^{2}+y^{2}\right) / a^{2}+\left(z^{2} / b^{2}\right) \leqslant 1 \\ 0, & \text { otherwise. }\end{cases}
$$

In the case of statistically homogeneous but anisotropic distributions of inclusions, the $\rho_{n}$ and, thus, the $S_{n}$ become functions of relative positions, i.e.,

$$
\begin{aligned}
\rho_{n}\left(\mathbf{r}_{1}, \mathbf{r}_{2}, \ldots, \mathbf{r}_{n}\right) & =\rho_{n}\left(\mathbf{r}_{12}, \ldots, \mathbf{r}_{1 n}\right), \\
& =\rho^{n} g_{n}\left(\mathbf{r}_{12}, \ldots, \mathbf{r}_{1 n}\right), \\
S_{n}\left(\mathbf{r}_{1}, \mathbf{r}_{2}, \ldots, \mathbf{r}_{n}\right) & =S_{n}\left(\mathbf{r}_{12}, \ldots, \mathbf{r}_{1 n}\right)
\end{aligned}
$$

The second equality of (2.5) defines the dimensionless $n$ particle distribution function $g_{n}$, where $\rho$ is the number density of spheroids. The one-point quantities are especially simple:

$$
\rho_{1}\left(\mathbf{r}_{1}\right)=\rho, \quad g_{1}\left(\mathbf{r}_{1}\right)=1, \quad S_{1}\left(\mathbf{r}_{1}\right)=\phi_{1} .
$$

For such media, it is useful to write (2.1) as

$$
S_{n}\left(\mathbf{r}_{12}, \ldots, \mathbf{r}_{1 n}\right)=1+\sum_{k=1}^{\infty}(-1)^{k} \rho^{k} S_{n}^{(k)}\left(\mathbf{r}_{12}, \ldots, \mathbf{r}_{1 n}\right),
$$

where

$$
\begin{aligned}
S_{n}^{(k)}= & \frac{1}{k !} \int g_{k}\left(\mathbf{r}_{r+1}, \ldots, \mathbf{r}_{n+k}\right) \\
& \times \prod_{j=n+1}^{n+k}\left(1-\prod_{i=1}^{n}\left[1-m\left(\mathbf{r}_{j i}\right)\right]\right) d \mathbf{r}_{j} .
\end{aligned}
$$

Our primary interest in (2.7) will be for the case of oriented spheroids of aspect ratio $\epsilon=b / a$

The task of computing the $S_{n}\left(\mathbf{r}^{n}\right)$ for oriented spheroids is made reasonably straightforward by exploiting an observation of Lebowitz and Perram. ${ }^{18}$ They studied the special case of equilibrium distributions of nonoverlapping (hard) spheroids, and noted that a scale transformation to coordinates

$$
\mathbf{R} \equiv(X, Y, Z)=(x, y,(a / b) z)
$$

converts spheroids of shape

$$
\left(x^{2}+y^{2}\right) / a^{2}+\left(z^{2} / b^{2}\right)=1
$$

and volume fraction $\phi_{2}$ into spheres of radius $a$ at the same volume fraction, thus reducing the thermodynamics and particle correlations of aligned hard spheroids to an equivalent problem involving hard spheres, a much studied model in the theory of liquids. ${ }^{19}$ Lado and Torquato ${ }^{17}$ recently employed this observation to compute the two-point function $S_{2}\left(\mathbf{r}_{12}\right)=S_{2}(r, \theta)$ for oriented hard spheroids for various values of $\epsilon$ and $\phi_{2}$, where $r_{12}=\left|\mathbf{r}_{12}\right|$ and $\theta$ is the polar angle between the $z$ axis and $\mathbf{r}_{12}$. They did so by showing that $S_{2}$ for this model scales as

$$
S_{2}\left(\mathbf{r}_{12} ; \epsilon\right)=S_{2}\left[\sigma_{0}\left(r_{12} / \sigma(\theta)\right) ; 1\right],
$$

where

$$
\sigma(\theta)=\frac{2 a}{\left[1-\left(1-a^{2} / b^{2}\right) \cos ^{2} \theta\right]^{1 / 2}}
$$

is an angle-dependent "sphere diameter" and $\sigma_{0} \equiv 2 a$ is the sphere diameter. Thus, (2.11) states that $S_{2}$ for hard spheroids of aspect ratio $\epsilon$ and inclusion volume fraction $\phi_{2}$ can be obtained from the $S_{2}$ for hard-sphere systems of volume fraction $\phi_{2}$ in which distances are scaled by $\sigma_{0}$. From (2.9) we extract the radial coordinate $R$ in the sphere system as

$$
R=2 a[r / \sigma(\theta)],
$$

where $\sigma(\theta)$ is given by $(2.12)$. 
We shall prove here that relation (2.11) applies not only to hard spheroids but to spheroids with an arbitrary degree of impenetrability, i.e., possibly overlapping spheroids. Moreover, it shall be demonstrated that a relation like (2.11) holds in the case of such spheroidal systems for $S_{n}$ for any value of $n$, i.e.,

$$
S_{n}\left(\mathbf{r}_{12}, \ldots, \mathbf{r}_{1 n} ; \epsilon\right)=S_{n}\left(\sigma_{0} \frac{r_{12}}{\sigma\left(\theta_{12}\right)}, \ldots, \sigma_{0} \frac{r_{1 n}}{\sigma\left(\theta_{1 n}\right)} ; 1\right) .
$$

We begin by considering the instance of $n=2$, i.e., $S_{2}\left(r_{12}\right)$. It is useful to review briefly the arguments of Lado and Torquato ${ }^{17}$ for the special case of hard spheroids. For such a model, the infinite series (2.7) truncates after the $n$th $\operatorname{term}^{12}$ (because of the presence of conflicting step functions) and for $n=2$ we have, after simplification,

$$
S_{2}\left(\mathbf{r}_{12} ; \epsilon\right)=1-\rho S_{2}^{(1)}\left(\mathbf{r}_{12} ; \epsilon\right)+\rho^{2} S_{2}^{(2)}\left(\mathbf{r}_{12} ; \epsilon\right),
$$

where

$$
\begin{aligned}
S_{2}^{(1)}\left(\mathbf{r}_{12} ; \epsilon\right) & \equiv V_{2}\left(\mathbf{r}_{12} ; \epsilon\right) \\
& =2 V_{1}(\epsilon)-\int m\left(\mathbf{r}_{13}\right) m\left(\mathbf{r}_{23}\right) d \mathbf{r}_{3}
\end{aligned}
$$

is the union volume of two aligned spheroids whose centroids are separated by $\mathbf{r}_{12}$,

$$
V_{1}(\epsilon)=(4 \pi / 3) a^{2} b=4 \pi a^{3} \epsilon / 3
$$

is the volume of one spheroid, and

$$
S_{2}^{(2)}\left(\mathbf{r}_{12} ; \epsilon\right)=\int g_{2}\left(\mathbf{r}_{34}\right) m\left(\mathbf{r}_{13}\right) m\left(\mathbf{r}_{24}\right) d \mathbf{r}_{3} d \mathbf{r}_{4} .
$$

For spheres of diameter $\sigma_{0}$, the intersection volume of two spheres is given by

$$
\begin{aligned}
V_{2}^{*}\left(R_{12} ; 1\right)= & \int m\left(R_{13}\right) m\left(R_{24}\right) d \mathbf{R}_{3} \\
= & \frac{4 \pi a^{3}}{3}\left[1-\frac{3}{2}\left(\frac{R_{12}}{\sigma_{0}}\right)+\frac{1}{2}\left(\frac{R_{12}}{\sigma_{0}}\right)^{3}\right] \\
& \times H\left(\sigma_{0}-R_{12}\right),
\end{aligned}
$$

where

$$
m(R)= \begin{cases}1, & R<a \\ 0, & R>a\end{cases}
$$

is the sphere indicator function and

$$
H(x)= \begin{cases}1, & x>0 \\ 0, & x<0\end{cases}
$$

is the Heaviside step function.

The coordinate transformation (2.9) in conjunction with (2.16) and (2.19) yields the union volume for spheroids as

$$
\begin{aligned}
S_{2}^{(1)}\left(\mathbf{r}_{12} ; \epsilon\right)= & 2 V_{1}(\epsilon)-\frac{b}{a} \int m\left(R_{13}\right) m\left(R_{23}\right) d \mathbf{R}_{3} \\
= & \frac{8}{3} \pi a^{2} b-\frac{4}{3} \pi a^{2} b\left[1-\frac{3}{2}\left(\frac{r_{12}}{\sigma(\theta)}\right)\right. \\
& \left.+\frac{1}{2}\left(\frac{r_{12}}{\sigma(\theta)}\right)^{3}\right] H\left(\sigma(\theta)-r_{12}\right)
\end{aligned}
$$

$$
=\frac{b}{a} S_{2}^{(1)}\left(\sigma_{0} \frac{r_{12}}{\sigma(\theta)} ; 1\right) .
$$

A simple scaled relationship like (2.22) for the twobody term $S_{2}^{(2)}$ is immediate from (2.18):

$$
\begin{aligned}
S_{2}^{(2)}\left(\mathbf{r}_{12} ; \epsilon\right) & =\left(\frac{b}{a}\right)^{2} \int g_{2}\left(R_{34}\right) m\left(R_{13}\right) m\left(R_{24}\right) d \mathbf{R}_{3} d \mathbf{R}_{4} \\
& =\left(\frac{b}{a}\right)^{2} S_{2}^{(2)}\left(R_{12} ; 1\right) \\
& =\left(\frac{b}{a}\right)^{2} S_{2}^{(2)}\left(\sigma_{0} \frac{r_{12}}{\sigma(\theta)} ; 1\right) .
\end{aligned}
$$

Note that since the volume fraction $\phi_{2}$ is invariant under the transformation (2.9), the number densities of the spheroid and sphere systems are related by the expression

$$
\rho(\epsilon)=(a / b) \rho(1) \text {. }
$$

Therefore, the combination of (2.15) and (2.22)-(2.24) yields the scaling relation (2.11) for hard inclusions.

Note that when $r_{12}=0,(2.15)$ yields the matrix volume fraction as

$$
S_{2}(0 ; \epsilon)=\phi_{1}=1-\eta
$$

where

$$
\eta=\rho(\epsilon) \frac{4}{3} \pi a^{2} b=\rho(1)_{3}^{4} \pi a^{3}
$$

is a reduced density and therefore, for hard particles, the inclusion volume fraction is simply related to the reduced density by

$$
\phi_{2}=\eta
$$

Now we shall prove the scaling relation (2.11) for the case of "fully penetrable" spheroids, i.e., spatially uncorrelated spheroids. The $n$-particle distribution functions $g_{n}$ are trivial for such a model:

$$
g_{n}\left(\mathbf{r}^{n}\right)=1, \quad \forall n \text {. }
$$

The series (2.7) for $n=2$ then yields

$$
S_{2}\left(\mathbf{r}_{12} ; \epsilon\right)=\exp \left[-\rho V_{2}\left(\mathbf{r}_{12} ; \epsilon\right)\right],
$$

implying that

$$
S_{2}^{(k)}\left(\mathbf{r}_{12} ; \epsilon\right)=\frac{\left[S_{2}^{(1)}\left(\mathbf{r}_{12} ; \epsilon\right)\right]^{k}}{k !}=\frac{\left[V_{2}\left(\mathbf{r}_{12} ; \epsilon\right)\right]^{k}}{k !}
$$

where $V_{2}$ is the union volume defined by (2.16). Use of (2.22) in (2.29) immediately proves (2.11). Again, the volume fractions of the spheroid and sphere systems are equal.

The matrix volume fraction $\phi_{1}$ for fully penetrable spheroids is easily obtained from (2.29) as follows:

$$
\phi_{1}=S_{2}(0 ; \epsilon)=\exp (-\eta),
$$

where $\eta$ is the reduced density defined in (2.26). Thus, because the inclusions are allowed to overlap, the inclusion volume fraction is given by

$$
\phi_{2}=1-\exp (-\eta)
$$

which is different from the totally impenetrable result (2.27).

Using similar arguments, it is easily proven that (2.11) 
applies to inclusions with an arbitrary degree of impenetrability. (An example of an interpenetrable-particle system is the penetrable-concentric-shell model. $)^{20}$ However, since the general proof of $(2.11)$ is essentially the same as the proof of the scaling relation (2.14) for $S_{n}$ for any $n$, we shall prove the latter. Let $\theta_{i j}$ be the polar angle that $\mathbf{r}_{i j}$ makes with the $z$ axis. Then the coordinate transformation (2.9) in conjunction with (2.8) gives

$$
\begin{aligned}
S_{n}^{(k)}\left(\mathbf{r}_{12}, \ldots, \mathbf{r}_{1 n} ; \epsilon\right) & =\left(\frac{b}{a}\right)^{k} \frac{1}{k !} \int g_{k}\left(R_{n+1, n+1}, \ldots, R_{n+2, n+1}\right) \prod_{j=n+1}^{n+k}\left(1-\prod_{i=1}^{n}\left[1-m\left(R_{j i}\right)\right]\right) d \mathbf{R}_{j} \\
& =\left(\frac{b}{a}\right)^{k} S_{n}^{(k)}\left(R_{12}, \ldots, R_{1 n} ; 1\right)=\left(\frac{b}{a}\right)^{k} S_{n}^{(k)}\left(\sigma_{0} \frac{r_{12}}{\sigma\left(\theta_{12}\right)}, \ldots, \sigma_{0} \frac{r_{1 n}}{\sigma\left(\theta_{1 n}\right)} ; 1\right) .
\end{aligned}
$$

Combination of (2.7) and (2.33) then gives the general scaling relation (2.14) that enables one to obtain the $S_{n}$ for oriented, possibly overlapping, spheroids with volume fraction $\phi_{2}$ and aspect ratio $\epsilon$ given the $S_{n}$ for the corresponding sphere system at the same volume fraction $\phi_{2}$. The relation (2.14) is valid for equilibrium as well as nonequilibrium ensembles of possibly overlapping spheroids.

We note in passing that (2.33) holds also in two dimensions for ellipses of shape

$$
\left(x^{2} / a^{2}\right)+\left(y^{2} / b^{2}\right)=1,
$$

oriented in the $y$ direction with semiaxes $a$ and $b$. Here $\sigma(\theta)$ is still given by (2.12) but $\theta$ is the angle between the $x$ axis and $\mathbf{r}$, and $a$ is the radius of the reference system of disks.

In the ensuing sections, the scaling relation (2.14) is employed to compute bounds on the trapping constant and effective thermal conductivity of suspensions of oriented spheroids as well as the coarseness for such a model. It shall generally be shown that the nontrivial dependence on volume fraction of these quantities is embodied in lower-order spatial moments of $\left[S_{2}(r)-\phi_{1}^{2}\right]$ for spheres of diameter $\sigma=2 a$, where $\phi_{1}^{2}$ is just the long-range value of $S_{2}(r)$. The two-point function has been computed for both equilibrium distributions of hard spheres ${ }^{13}$ and fully overlapping (penetrable) spheres. ${ }^{21}$ Thus, the spatial moments are easily evaluated for these two models and in Table I we tabulate the zeroth, first, and second moments for both impenetrable and fully penetrable spheres, where the general moment is defined by

TABLE I. Lower-order dimensionless spatial moments $\left\langle x^{n}\right\rangle_{0}$ as defined by (2.35) as a function of the particle volume fraction $\phi_{2}$ for two spherical

\begin{tabular}{|c|c|c|c|c|c|c|}
\hline & Totally & penetra & pheres & Full & netrabl & leres \\
\hline$\phi_{2}$ & $\left.x^{0}\right\rangle_{0} x$ & \rangle$_{0} x$ & $\left.{ }^{2}\right\rangle_{0}>$ & $\left.x^{0}\right\rangle_{0}$ & $\left.\varepsilon^{1}\right\rangle$ & $\left.{ }^{2}\right\rangle_{0} \times 10$ \\
\hline 0.1 & 29.48 & 6.420 & 1.904 & 33.09 & 8.74 & 3.617 \\
\hline 0.2 & 45.84 & 8.280 & 1.769 & 57.50 & 15.01 & 6.173 \\
\hline 0.3 & 52.40 & 7.922 & 1.220 & 73.55 & 18.94 & 7.726 \\
\hline 0.4 & 51.70 & 6.552 & 0.718 & 81.55 & 20.66 & 8.349 \\
\hline 0.5 & 45.78 & 4.822 & 0.366 & 81.90 & 20.34 & 8.124 \\
\hline 0.6 & 36.20 & 2.048 & 0.156 & 75.10 & 18.19 & 7.157 \\
\hline 0.7 & & & & 61.85 & 14.49 & 5.585 \\
\hline 0.8 & & & & 43.21 & 9.62 & 3.596 \\
\hline 0.9 & & & & 20.88 & 4.24 & 1.494 \\
\hline
\end{tabular}
models: totally impenetrable (hard) and fully penetrable (overlapping) spheres.

$$
\left\langle x^{n}\right\rangle_{0}=\int_{0}^{\infty} x^{n}\left[S_{2}(x)-\phi_{1}^{2}\right] d x .
$$

Here $x=r / 2 a$ is a dimensionless distance and the brackets with subscript " 0 " shall denote an average in a reference system of spheres. Results for hard spheres were computed using the accurate Verlet-Weis ${ }^{22}$ fit for the radial distribution function. The volume-fraction value $\phi_{2}=0.6$ for hard spheres corresponds to approximately $95 \%$ of the random close-packing value. ${ }^{23}$

For the case of arbitrary $\epsilon$, the generalized moments

$$
M_{n}(\epsilon) \equiv \frac{1}{4 \pi} \int d \mathbf{r} r^{n-2}\left[S_{2}(\mathbf{r}, \epsilon)-\phi_{1}^{2}\right]
$$

are simplified by the scaling relation (2.14) to read

$$
M_{n}(\epsilon)=(2 a)^{n}\left\langle x^{n}\right\rangle_{0} \xi_{n}(\epsilon)
$$

where

$$
\xi_{n}(\epsilon) \equiv \frac{1}{2} \int_{-1}^{1} d u \frac{1}{\left[1-\left(1-1 / \epsilon^{2}\right) u^{2}\right]^{(n+1) / 2}}
$$

is a purely shape-dependent factor. The first three such factors are explicitly

$$
\begin{aligned}
& \xi_{0}(\epsilon)=\left\{\begin{array}{l}
\frac{1}{2 \chi_{\mathrm{a}}} \ln \frac{1+\epsilon \chi_{a}}{1-\epsilon \chi_{a}}, \epsilon<1, \\
\frac{1}{\chi_{b}} \sin ^{-1} \chi_{b}, \epsilon>1,
\end{array}\right. \\
& \xi_{1}(\epsilon)=\left\{\begin{array}{l}
\frac{1}{\chi_{a}} \tan ^{-1} \chi_{a}, \epsilon<1, \\
\frac{1}{2 \chi_{b}} \ln \frac{1+\chi_{b}}{1-\chi_{b}}, \epsilon>1,
\end{array}\right. \\
& \xi_{2}(\epsilon)=\epsilon, \text { all } \epsilon,
\end{aligned}
$$

where

$$
\chi_{a}^{2}=-\chi_{b}^{2}=\left(a^{2} / b^{2}\right)-1 .
$$

\section{BOUNDS ON THE DIFFUSION-CONTROLLED TRAPPING CONSTANT}

Consider the problem of diffusion-controlled reactions in a two-phase random medium composed of a trap-free region of volume fraction $\phi_{1}$ containing reactive particles and a trap region of volume fraction $\phi_{2}$. Reactant diffuses in the trap-free region but is instantly absorbed on contact with any trap. At steady state, the rate of production of the diffusing 
species is exactly compensated by its removal by the traps. The trapping constant $\gamma$ is proportional to the trapping rate. $^{24}$

Virtually all previous calculations of $\gamma$ for continuum models have been carried out for isotropic distributions of spherical traps. ${ }^{25}$ There is a dearth of results for statistically anisotropic models. A recent study of $\gamma$ for the special case of oriented, slender, rodlike traps at dilute concentrations ${ }^{26}$ stands as an exception. To our knowledge, $\gamma$ has not been computed for any anisotropic model at high trap concentrations where there is strong competition between the traps.

A useful means of estimating $\gamma$ at arbitrary trap volume fraction is by the determination of rigorous bounds on $\gamma$. Bounds on effective properties of random media generally capture the salient features of the problem and can provide an accurate prediction of the property for a wide range of conditions. $^{3}$

Torquato and Rubinstein ${ }^{8}$ derived a two-point lower bound on $\gamma$, termed a "void" bound, that is valid for any statistically anisotropic medium ${ }^{27}$ and is given by

$$
\gamma \geqslant \gamma_{L}^{(2)}
$$

where

$$
\gamma_{L}^{(2)}=\left(\frac{1}{4 \pi \phi_{2}^{2}} \int \frac{1}{r}\left[S_{2}(\mathbf{r})-\phi_{1}^{2}\right] d \mathbf{r}\right)^{-1} .
$$

For future discussions it is useful to distinguish between statistical anisotropy and macroscopic anisotropy. ${ }^{24}$ By statistical anisotropy we mean that the $n$-point functions $S_{n}$ do not remain invariant under rotation. Macroscopic anisotropy refers to anisotropy with respect to the macroscopic properties of the system, e.g., effective conductivity tensor $\boldsymbol{\sigma}_{e}$. Although macroscopic anisotropy always implies statistical anisotropy, the converse is not necessarily true. For example, a cubic array of spheres (statistically anisotropic) is described by a scalar effective conductivity $\sigma_{c}$; similarly a medium composed of oriented spheroids (statistically anisotropic), though described by an effective conductivity tensor $\sigma_{e}$, possesses a scalar trapping constant $\gamma$. Indeed, the trapping constant $\gamma$ is a scalar for any statistically anisotropic medium.

Consider the evaluation of the void bound (3.1) for distributions of oriented, possibly overlapping, spheroidal traps of aspect ratio $\epsilon=b / a$. The scaling relation (2.11) in combination with (3.2) yields

$$
\gamma_{L}^{(2)}(\epsilon)=\left(\phi_{2}^{2} / 4 a^{2}\langle x\rangle_{\mathrm{o}}\right) f(\epsilon)
$$

where

$$
f(\epsilon)=1 / \xi_{1}(\epsilon),
$$

$\xi_{1}(\epsilon)$ is given by (2.40), and $\langle x\rangle_{0}$ is the first spatial moment for spheres defined by (2.35). Thus, the nontrivial volumefraction dependence enters the bound only through $\langle x\rangle_{0}$, which is tabulated in Table I for hard (i.e., totally impenetrable) spheres and fully penetrable spheres, respectively. The shape-dependent function $f(b / a)$ is shown in Fig. 1. For very slenderinclusions $(b \gg a), f(b / a) \sim[\ln (2 b / a)]^{-1}$, and for disklike inclusions $(b \ll a), f(b / a) \sim 2 a / \pi b$.

It is noteworthy that the two-point bound (3.3) gives the following symmetrical relation between spheroids of as-

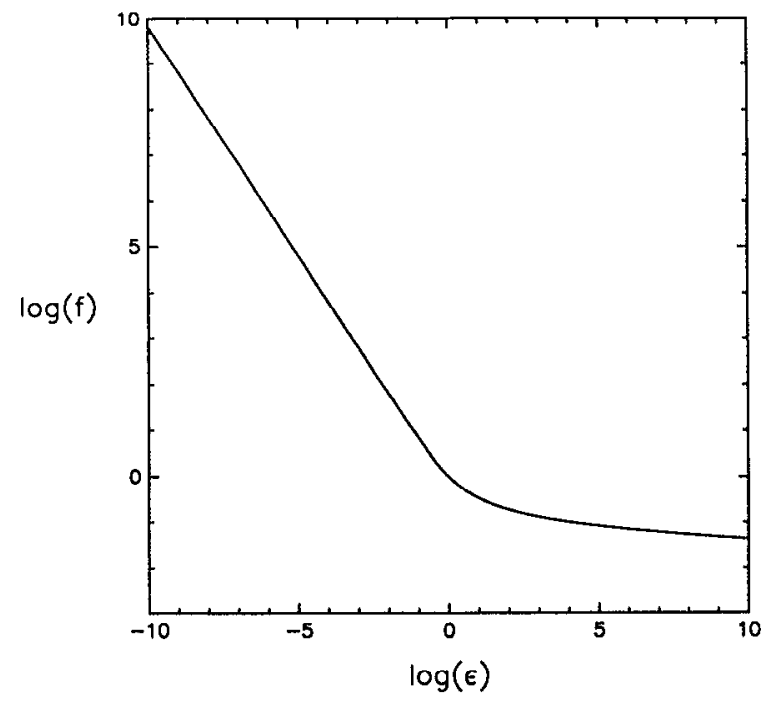

FIG. 1. Log-log plot of the shape-dependent function $f(\epsilon)$, defined by (3.4), versus the aspect ratio $\epsilon$.

pect ratio $\epsilon$ and those of aspect ratio $\epsilon^{-1}$ at the same volume fraction $\phi_{2}$ :

$$
\frac{\gamma_{L}^{(2)}(\epsilon)}{\gamma_{L}^{(2)}(1 / \epsilon)}=\frac{f(\epsilon)}{f(1 / \epsilon)} .
$$

Note that the right-hand side of (3.5) is only shape-dependent (i.e., independent of $\phi_{2}$ ). Thus, results for $\gamma_{L}^{(2)}(\epsilon)$ can be obtained from results for $\gamma_{L}^{(2)}(1 / \epsilon)$ by multiplying the latter by a shape-dependent function. Relation (3.5) suggests that the actual trapping constant for spheroids obeys a similar simple scaling law for a certain region of the parameter space.

For the case of infinitely dilute spheroids, we can evaluate the void bound (3.3) and compare it with the exact result

$$
\gamma_{s}(\epsilon)=\left(3 \phi_{2} / a^{2}\right) f(\epsilon)
$$

which for spheres $(\epsilon=1)$ reduces to the well-known Smoluchowski result. Result (3.6) is easily obtained given the solution of the boundary-value problem for an isolated, perfectly absorbing spheroidal trap in an infinite medium. Note that (3.6) depends on the same shape-dependent function $f(\epsilon)$ which arises in the bound (3.3). In this infinitely dilute limit, Torquato and Rubinstein ${ }^{8}$ showed that

$$
\langle x\rangle_{\circ} \sim \phi_{2} / 10
$$

so that

$$
\gamma_{L}^{(2)}(\epsilon)=\left(5 / 2 a^{2}\right) f(\epsilon) \quad\left(\phi_{2} \ll 1\right) .
$$

Thus, for $\phi_{2} \ll 1$, the bound gives $5 \gamma_{s}(\epsilon) / 6$. Observe that the two-point bound (3.3) and the low-density result (3.8) gives another scaling relation between spheroids of aspect ratio $\epsilon$ and those of aspect ratio $1 / \epsilon$ :

$$
\frac{\gamma_{L}^{(2)}(\epsilon)}{\gamma_{s}(\epsilon)}=\frac{\gamma_{I}^{(2)}(1 / \epsilon)}{\gamma_{s}(1 / \epsilon)}
$$

The scaled trapping constants of relation (3.9) are functions of volume fraction only, specifically, they equal $\phi_{2}\left[12\langle x\rangle_{0}\right]^{-1}$. It is expected that the true trapping constant will behave in a similar fashion for small $\phi_{2}$ and $b / a$ not 
not much different from unity, but generally such a scaling will depend upon the aspect ratio as well.

Figures 2 and 3 show the lower bounds (3.3) on the dimensionless trapping constant $\gamma a^{2}$ as a function of the aspect ratio $b / a$ at $\phi_{2}=0.2$ and 0.6 , respectively, for hard and overlapping spheroids. The bounds are seen to be monotonically decreasing functions of $b / a$, as expected. For fixed $b / a$, the hard spheroid results are always greater than the overlapping spheroid results, the difference widening as $\phi_{2}$ increases. This behavior is due to the fact that distributions of hard spheroidal traps have more surface area available for reaction than overlapping spheroidal traps at the same volume fraction.

Figures 4 and 5 depict the lower bounds on $\gamma a^{2}$ versus the trap volume fraction $\phi_{2}$ for $b / a=5$ and 0.2 , respectively, for hard and overlapping spheroids. Not surprisingly, these results indicate that the trapping constant increases with increasing trap concentration. The symmetrical scaling relation (3.5) is graphically illustrated in these figures.

In Fig. 6 we plot lower bounds on the trapping constant scaled by the dilute-limit value, $\gamma / \gamma_{s}$, as a function of $\phi_{2}$. By the scaling relation (3.9) such a result is independent of the aspect ratio and therefore applies to all aspect ratios.

\section{BOUNDS ON THE EFFECTIVE CONDUCTIVITY TENSOR}

Sen and Torquato ${ }^{7}$ have recently derived two-, three-, and four-point bounds on the effective conductivity tensor $\sigma_{e}$ for general $d$-dimensional two-phase media with phase conductivities $\sigma_{1}$ and $\sigma_{2}$. The $n$-point bounds have been shown to depend explicitly upon functionals of the $n$-point probability functions $S_{1}, S_{2}, \ldots, S_{n}$ for two-phase statistically anisotropic media of arbitrary microstructure. These functionals have been related" to the "normalization factors" and "weights" of Milton ${ }^{2}$ and thus the latter have been explicitly given in terms of the $S_{n}$ for the first time.

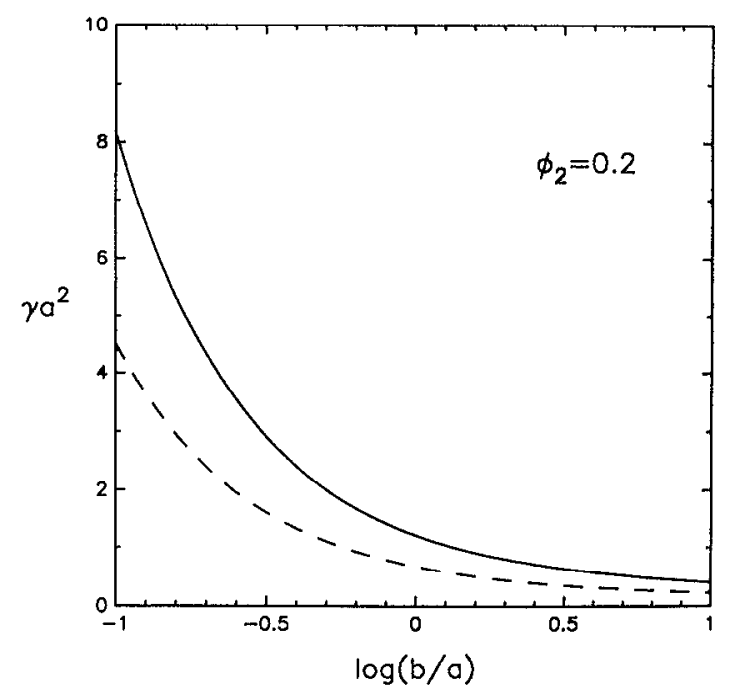

FIG. 2. Two-point lower bounds (3.3) on the dimensionless trapping constant $\gamma a^{2}$ as a function of the log of the aspect ratio $b / a$ at the trap volume fraction $\phi_{2}=0.2$ for hard $(\longrightarrow)$ and overlapping ( $-\longrightarrow$ ) oriented spheroids.

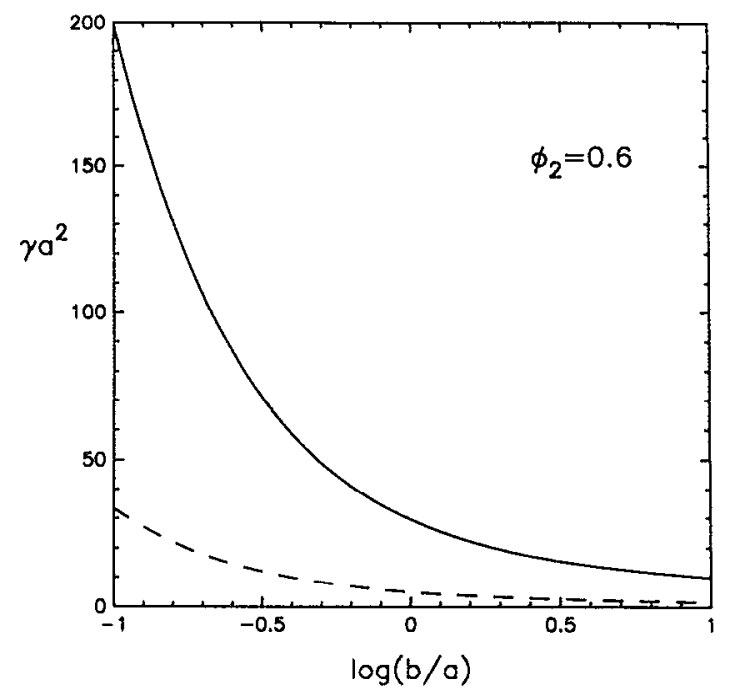

FIG. 3. As in Fig. 2, with $\phi_{2}=0.6$.

We are concerned here with the two-point Sen-Torquato bounds which, for $\sigma_{2} \geqslant \sigma_{1}$, are given by

$$
\begin{aligned}
& \boldsymbol{\sigma}_{L}^{(2)} \leqslant \boldsymbol{\sigma}_{e} \leqslant \boldsymbol{\sigma}_{U}^{(2)} \\
& \frac{\boldsymbol{\sigma}_{L}^{(2)}}{\sigma_{1}}= {\left[U+\left(\phi_{2} U-\frac{1}{\phi_{2}} a_{2}\right) \frac{\left(\sigma_{2}-\sigma_{1}\right)}{\sigma_{1}}\right] } \\
& \cdot\left(U-\frac{1}{\phi_{2}} a_{2} \frac{\left(\sigma_{2}-\sigma_{1}\right)}{\sigma_{1}}\right)^{-1} \\
& \frac{\boldsymbol{\sigma}_{U}^{(2)}}{\sigma_{2}}= {\left[U+\left(\phi_{1} U-\frac{1}{\phi_{1}} a_{2}\right) \frac{\left(\sigma_{1}-\sigma_{2}\right)}{\sigma_{2}}\right] } \\
& \cdot\left(U-\frac{1}{\phi_{1}} a_{2} \frac{\left(\sigma_{1}-\sigma_{2}\right)}{\sigma_{2}}\right)^{-1},
\end{aligned}
$$

where

$$
a_{2}=-\phi_{1} \phi_{2} A_{2}^{*}
$$

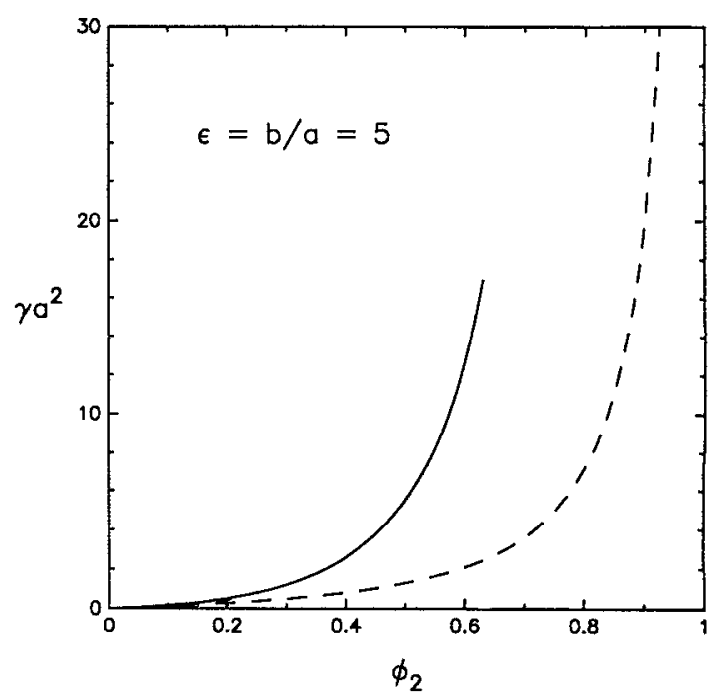

FIG. 4. Two-point lower bounds (3.3) on the dimensionless trapping constant $\gamma a^{2}$ versus the trap volume fraction $\phi_{2}$ for hard $(-)$ and overlapping $(--\rightarrow$ oriented, prolate spheroids with an aspect ratio $b / a=5$. 


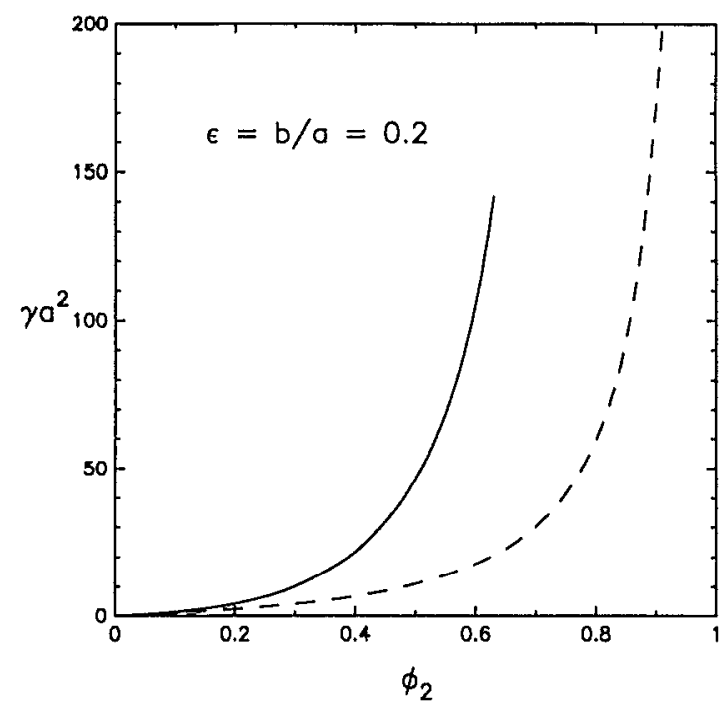

FIG. 5. As in Fig. 2, for oblate spheroids with an aspect ratio $b / a=0.2$.

$\mathrm{A}_{2}^{*}=\frac{U}{d}-\frac{d}{2 \pi(d-1) \phi_{1} \phi_{2}} \int_{\delta}\left(\frac{\mathbf{r r} d-r^{2} U}{r^{d+2}}\right)\left[S_{2}(\mathbf{r})-\phi_{1}^{2}\right]$

Here $\mathrm{U}$ is the identity tensor and the subscript $\delta$ on the integral of (4.4) indicates that the integration is to be carried out with the exclusion of an infinitesimally small $d$-dimensional sphere centered at $r$. The notation of (4.1) signifies that the tensors $\left(\sigma_{U}^{(2)}-\sigma_{e}\right)$ and $\left(\sigma_{e}-\sigma_{L}^{(2)}\right)$ are positive-semidefinite. The two-point parameters $a_{2}$ and $A_{2}^{*}$ have the following properties. ${ }^{7}$ First, for macroscopically isotropic media,

$$
\mathrm{d}_{2}=-\phi_{1} \phi_{2} \mathrm{U} / d, \quad \mathrm{~A}_{2}^{*}=\mathrm{U} / d \text {. }
$$

Second, for general isotropic media the traces of these tensors are given by

$$
\operatorname{tr}\left(a_{2}\right)=-\phi_{1} \phi_{2}, \quad \operatorname{tr}\left(A_{2}^{*}\right)=0 .
$$

Third, the two-point parameters are bounded from above and below as follows:

$$
\begin{aligned}
& -\phi_{1} \phi_{2} \leqslant\left(a_{2}\right)_{k k} \leqslant 0 \\
& 0 \leqslant\left(A_{2}^{*}\right)_{k k} \leqslant 1,
\end{aligned}
$$

where $X_{k k}(k=1, \ldots, d)$ denote the diagonal elements of a tensor $X$. The two-point bounds (4.1) are the anisotropic generalization of the isotropic Hashin-Shtrikman bounds. ${ }^{28}$ Note that unlike the Hashin-Shtrikman bounds, the anisotropic bounds (4.1) generally depend upon $S_{2}$ in a nontrivial manner.

For three-dimensional distributions of inclusions aligned in the $x_{3}$ direction which possess transverse isotropy and azimuthal symmetry (e.g., circular cylinders and spheroids), the so-called "polarization" tensor $A_{2}^{*}$ becomes

$$
\mathrm{A}_{2}^{*}=\left[\begin{array}{ccc}
Q & 0 & 0 \\
0 & Q & 0 \\
0 & 0 & 1-2 Q
\end{array}\right],
$$

where

$$
\begin{aligned}
Q= & \frac{1}{3}-\lim _{\delta-0} \frac{1}{2 \phi_{1} \phi_{2}} \int_{\delta}^{\infty} \frac{d r}{r} \int_{0}^{\pi} d(\cos \theta) \\
& \times P_{2}(\cos \theta)\left[S_{2}(\mathbf{r})-\phi_{1}^{2}\right] .
\end{aligned}
$$

Here, $P_{2}(x)$ is the Legendre polynomial of order two.

Consider evaluating the key integral $(4.10)$ for possibly overlapping, oriented spheroids of aspect ratio $\epsilon$. This shall be accomplished by again invoking the scaling relation (2.11). Let

$$
K=\lim _{\delta \rightarrow 0} \int_{\delta}^{\infty} \frac{d r}{r} \int_{-1}^{1} d u P_{2}(u)\left[S_{2}(\mathrm{r}, \epsilon)-\phi_{1}^{2}\right]
$$

with $u=\cos \theta$. Substitution of relation (2.11) into (4.11) yields

$$
\begin{aligned}
K & =\lim _{\delta \rightarrow 0} \int_{\delta}^{\infty} \frac{d r}{r} \int_{-1}^{1} d u P_{2}(u)\left[S_{2}\left(\frac{r}{\sigma(u)} ; 1\right)-\phi_{1}^{2}\right]=\lim _{\delta \rightarrow 0}-\frac{1}{2} \int_{\delta}^{\infty} \frac{d r}{r} \int_{-1}^{1} d u\left(\frac{d}{d u}\left[u\left(1-u^{2}\right)\right]\right)\left[S_{2}\left(\frac{r}{\sigma(u)} ; 1\right)-\phi_{1}^{2}\right] \\
& =\frac{1}{2} \int_{0}^{\infty} d r \int_{-1}^{1} d u u\left(1-u^{2}\right) S_{2}^{\prime}\left(\frac{r}{\sigma(u)} ; 1\right) \frac{d}{d u}\left(\frac{1}{\sigma(u)}\right) \\
& =-\frac{1}{2}\left[S_{2}(0 ; 1)-\phi_{1}^{2}\right] \int_{-1}^{1} d u u\left(1-u^{2}\right) \sigma(u) \frac{d}{d u}\left(\frac{1}{\sigma(u)}\right) .
\end{aligned}
$$

where prime denotes total derivative. Note that $K$, unlike the other key functionals in this paper, depends upon $S_{2}-\phi_{1}^{2}$ in a trivial way. Substitution of (4.12) into (4.10) finally gives

$$
Q=\frac{1}{3}+\frac{1}{4} \int_{-1}^{1} d u u\left(1-u^{2}\right) \sigma(u) \frac{d}{d u}\left(\frac{1}{\sigma(u)}\right),
$$

showing that $Q$ interestingly is a purely shape-dependent parameter independent of the structure of the spheroidal distribution and of the volume fraction. [Note that $\sigma(u)$ in (4.12) and (4.13) is the angle-dependent "diameter" and should not be confused with the phase conductivities $\sigma_{i}$.] The integral of (4.13) is easily evaluated with the result

$$
\begin{aligned}
Q= & \frac{1}{2}\left\{1+\frac{1}{(b / a)^{2}-1}\left[1-\frac{1}{2 \chi_{b}} \ln \left(\frac{1+\chi_{b}}{1-\chi_{b}}\right)\right]\right\}, \\
& \frac{b}{a}>1 \\
Q= & \frac{1}{2}\left[1+\frac{1}{(b / a)^{2}-1}\left(1-\frac{1}{\chi_{a}} \tan ^{-1}\left(\chi_{a}\right)\right)\right] \\
& \frac{b}{a}<1
\end{aligned}
$$




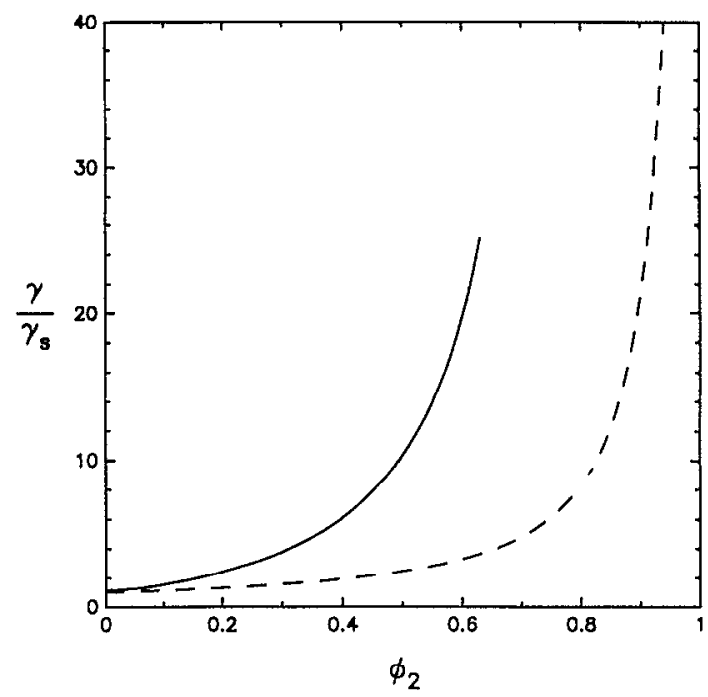

FIG. 6. Two-point lower bounds (3.3) on the scaled trapping constant $\gamma / \gamma$, as a function of the trap volume fraction for hard (- - ) and overlapping $(---)$ oriented spheroids of arbitrary aspect ratio $\epsilon$. Note that this figure illustrates the scaling relation $\gamma_{L}^{(2)}(\epsilon) / \gamma_{s}(\epsilon)=\gamma_{L}^{(2)}(1 / \epsilon) / \gamma_{s}(1 / \epsilon)$ given by (3.10).

where $\chi_{a}$ and $\chi_{b}$ are defined by (2.42). $Q=1 / 3,1 / 2$, and 0 for flat disks $(\epsilon=0)$, spheres $(\epsilon=1)$ and needles $(\epsilon=\infty)$, respectively. The parameter $Q$ is plotted in Fig. 7.

Willis $^{29}$ actually was the first to obtain these results for spheroids using a completely different approach. He did so, elegantly, without explicitly evaluating the integral $K$ involving $S_{2}$ by employing a well-known result due to Eshelby for ellipsoids. ${ }^{30}$ Thus, he did not note or use the scaling relation (2.11). The nature of Willis' derivation, however, prevented him from drawing what we believe to be an important conclusion, namely, the two-point bounds for spheroids are insensitive to the type of spatial correlations between the spheroids, i.e., one gets the same answer whether the spheroids are penetrable or not. This is not true for inclusions of

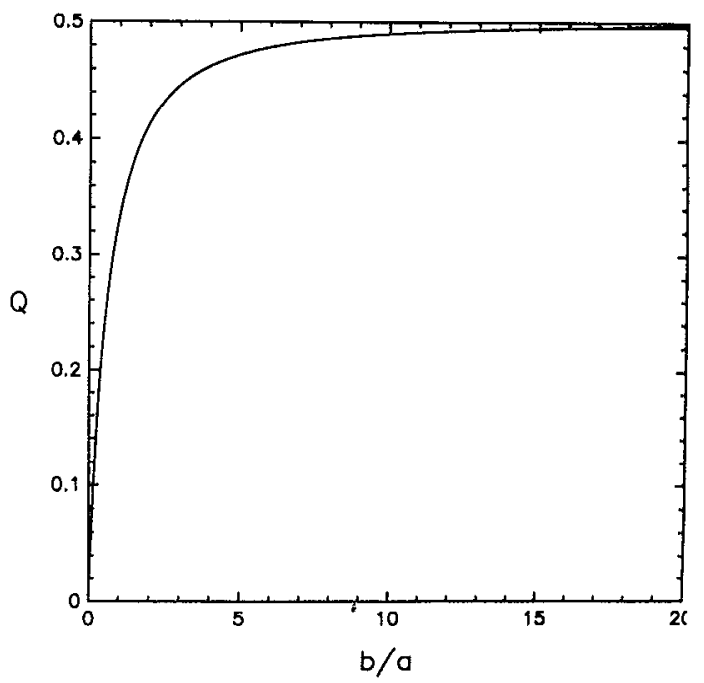

FIG. 7. Shape-dependent function $Q$, given by (4.14) and (4.15), versus the aspect ratio $b / a$ for possibly overlapping spheroids. arbitrary shape. Torquato and Sen, ${ }^{7}$ for example, evaluated the two-point bounds (4.1) for oriented, overlapping cylinders of finite aspect ratio and found some sensitivity due to structure.

Figure 8 depicts the two-point bounds on the three diagonal components of the effective conductivity tensor $\left(\sigma_{e}\right)_{i i}$ ( $i=1,2$, and 3 ) for conducting ( $\alpha=\sigma_{2} / \sigma_{1}=10$ ), oriented, slender fibers using the spheroidal results for $b / a=10$. Figure 9 shows the corresponding bounds for the case of insulating $(\alpha=0.1)$, oriented, penny-shaped cracks $(b / a=0.1)$. Again, we emphasize that these results apply to spheroidal systems with an arbitrary degree of impenetrability.

\section{COARSENESS: LOCAL VOLUME FRACTION FLUCTUATIONS}

Although the volume fraction is constant for statistically homogeneous but anisotropic media, on a spatially local level it fluctuates. An interesting and relatively unexplored question in the study of multiphase random media is the following: How does the "local" volume fraction fluctuate about its average value? The answer to this query has relevance to a number of problems, including scattering by heterogeneous media, ${ }^{31}$ transport through composites and porous media, ${ }^{1-3}$ and the study of noise and granularity of photographic images. ${ }^{16}$

The local volume fraction $\tau(\mathbf{x})$ at some position $\mathbf{x}$ is defined to be the volume fraction in one of the phases, say phase 1 , contained in some generally finite-sized "observation region" (see Fig. 10). Clearly, $\tau(x)$ is a random variable and becomes a constant equal to $\phi_{1}$ in the limit of an infinitely large observation region. Lu and Torquato ${ }^{16}$ have termed the coarseness, $C$, to be the standard deviation associated with $\tau(\mathrm{x})$ divided by $\phi_{1}$, i.e.,

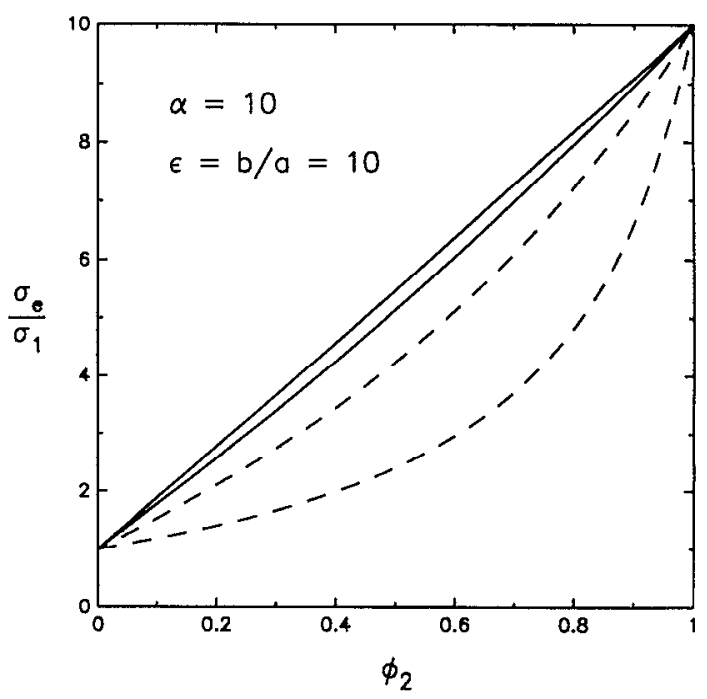

FIG. 8. Two-point Sen-Torquato bounds' on the dimensionless effective conductivity diagonal components $\sigma_{e} / \sigma_{1}\left[\sigma_{e} \equiv\left(\sigma_{e}\right)_{i i}\right]$ versus the inclusion volume fraction $\phi_{2}$ for a composite containing conducting ( $\alpha=\sigma_{2} /$ $\left.\sigma_{2}=10\right)$, oriented, slender $(b / a=10)$, spheroidal inclusions. The dashed lines are bounds for $\left(\sigma_{e}\right)_{11}=\left(\sigma_{e}\right)_{22}$ and the solid lines are bounds for $\left(\sigma_{e}\right)_{33}$. 


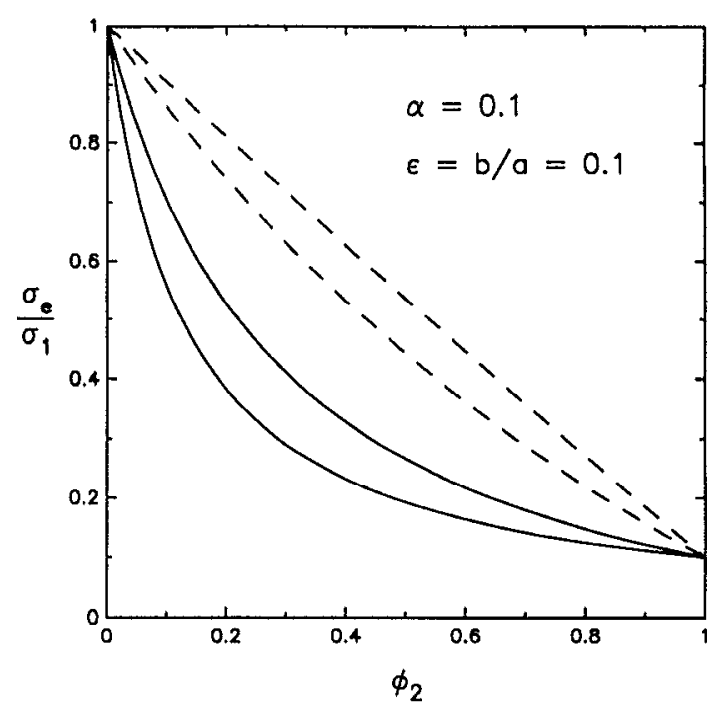

FIG. 9. As in Fig. 7, for nonconducting ( $\alpha=\sigma_{2} / \sigma_{1}=0.1$ ), oriented, flat $(b / a=0.1)$ oblate spheroidal inclusions.

$$
\begin{aligned}
C & =\sqrt{\left(\left\langle\tau^{2}\right\rangle-\langle\tau\rangle^{2}\right)} / \phi_{1} \\
& =\sqrt{\left(\left\langle\tau^{2}\right\rangle-\phi_{1}^{2}\right)} / \phi_{1},
\end{aligned}
$$

where angular brackets denote an ensemble average. For infinitely large and infinitesimally small observation regions, $C$ is, respectively, given by

$$
C=0
$$

and

$$
C=\sqrt{\phi_{1} \phi_{2}} / \phi_{1}
$$

The coarseness provides a quantitative measure of nonuniformity of coverage of the phases.

Lu and Torquato ${ }^{16}$ obtained a general expression for the coarseness for arbitrary, statistically anisotropic, two-phase media in terms of $S_{2}(r)$. They were the first to study the effect of exclusion volume on $C$ for arbitrary observation region sizes by computing it for $d$-dimensional hard spheres.

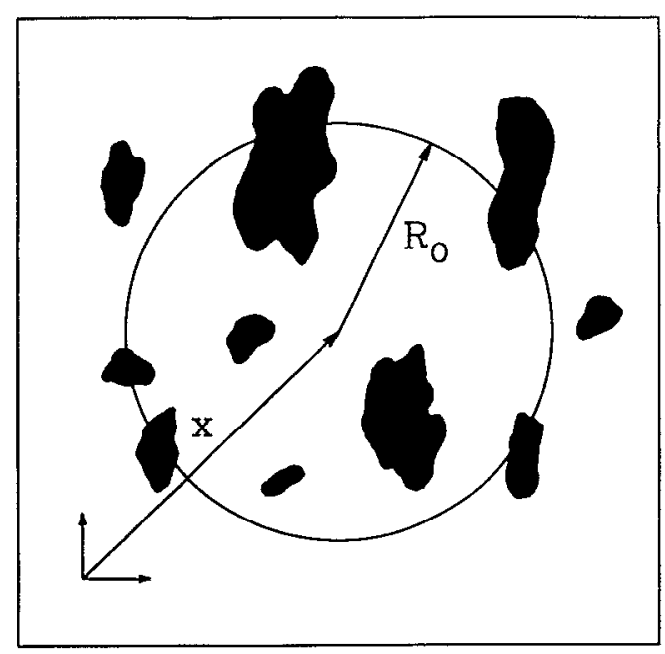

FIG. 10. Schematic of two-dimensional two-phase media with a circular observation region of radius $R_{0}$ centered at position $\mathrm{x}$.

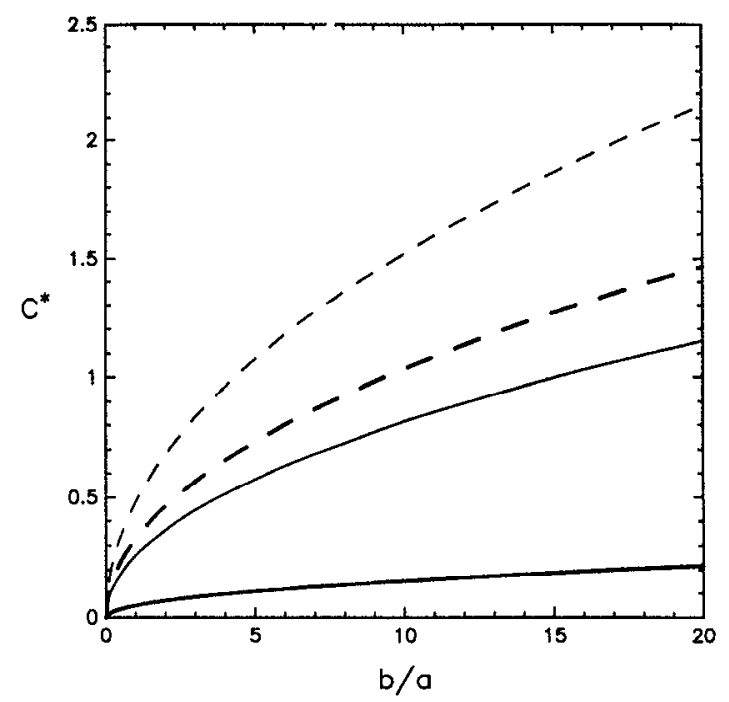

FIG. 11. Scaled coarseness $C^{*}=C\left[V_{0} / V_{1}(1)\right]^{1 / 2}$ versus the aspect ratio $b / a$ for hard (-) and overlapping ( $-\ldots$ ) oriented spheroids. Lighter lines correspond to an inclusion volume fraction of $\phi_{2}=0.2$. Heavier lines correspond to $\phi_{2}=0.6 . V_{1}(1)=4 \pi a^{3} / 3$.

The coarseness has heretofore not been computed for statistically anisotropic distributions of inclusions.

We shall compute the coarseness of distributions of oriented, possibly overlapping, spheroids in cases in which the characteristic size of the observation region is much larger than the spatial correlation length, since the coarseness relation is then somewhat simplified. ${ }^{16}$ In practice, the size of the observation region is, in fact, much larger than the characteristic size of the particles. For general media and very large observation region, it can be shown that ${ }^{16}$

$$
C=\frac{1}{\phi_{1} V_{o}}\left(\int\left[S_{2}(\mathbf{r})-\phi_{1}^{2}\right] d \mathbf{r}\right)^{1 / 2},
$$

where $V_{O}$ is the volume of the observation region. Use of the scaling relation $(2.11)$ in (5.4) yields the desired result for spheroids, namely,

$$
\begin{aligned}
C & =\left(2 \sqrt{6} / \phi_{1}\right)\left[V_{1}(\epsilon) / V_{o}\right]^{1 / 2}\left\langle x^{2}\right\rangle_{o}^{1 / 2}, \\
& =4 \sqrt{2}\left(a^{3} \epsilon / V_{o}\right)^{1 / 2}\left\langle x^{2}\right\rangle_{o}^{1 / 2},
\end{aligned}
$$

where $V_{1}(\epsilon)$ is the volume of a spheroid as given by $(2.17)$ and $\left\langle x^{2}\right\rangle_{\circ}$ is the second spatial moment for spheres defined by (2.35). Here we have used (2.41). Thus, $C$, for fixed semiaxis $a$, observation region size and volume fraction $\phi_{2}$, is proportional to the square root of the aspect ratio $\epsilon$.

Figure 11 shows the scaled coarseness $C^{*}=C\left[V_{O} /\right.$ $\left.V_{1}(1)\right]^{1 / 2}$ as a function of aspect ratio $b / a$ for both hard and overlapping spheroids, where $V_{1}(1)=4 \pi a^{3} / 3$ is just the volumc of a sphere. Here we have used the results of the second spatial moment $\left\langle x^{2}\right\rangle_{\circ}$ given in Table I. As was found in the case of spheres, ${ }^{16}$ exclusion volume effects lower the coarseness.

Finally, we note that the coarseness $C(\epsilon)$ for spheroids of aspect ratio $\epsilon$ is related to the same quantity for spheres $C(1)$ by the simple relation

$$
C(\epsilon) / C(1)=\sqrt{\epsilon} \text {. }
$$




\section{ACKNOWLEDGMENTS}

S. Torquato gratefully acknowledges the partial support of the Office of Basic Energy Sciences, U. S. Department of Energy, under Grant No. DEFG05-86ER 13482 and the Air Force Office of Scientific Research under Grant No. 900090 .

'Z. Hashin, J. Appl. Mech. 50, 481 (1983).

${ }^{2}$ G. W. Milton, Commun. Math. Phys. 111, 281 (1987).

${ }^{3}$ S. Torquato, Appl. Mech. Rev. 44, 37 (1991).

${ }^{4}$ S. Torquato, J. Stat. Phys. 45, 843 (1986); Phys. Rev. B 35, 5385 (1987).

${ }^{5}$ G. W. Milton, Phys. Rev. Lett. 46, 542 (1981).

${ }^{6}$ S. Torquato, J. Appl. Phys. 58, 3790 (1985).

${ }^{7}$ A. K. Sen and S. Torquato, Phys. Rev. B 39, 4504 (1989); S. Torquato and

A. K. Sen, J. Appl. Phys. 67, 1145 (1990).

${ }^{8}$ S. Torquato and J. Rubinstein, J. Chem. Phys. 90, 1644 (1989).

'S. Prager, Phys. Fluids 4, 1477 ( 1961 ); J. G. Berryman and G. W. Milton,

J. Chem. Phys. 83, 754 (1985).

${ }^{10} \mathrm{~J}$. Rubinstein and S. Torquato, J. Fluid Mech. 206, 25 (1989).

${ }^{11}$ G. W. Milton and N. Phan-Thien, Proc. R. Soc. London Ser. A 380, 305 (1989).

${ }^{12}$ S. Torquato and G. Stell, J. Chem. Phys. 77, 2071 (1982).

${ }^{13}$ S. Torquato and G. Stell, J. Chem. Phys. 82, 980 (1985).

${ }^{14} \mathrm{See}$ also numerous references contained in Ref. 3.
${ }^{15}$ S. Torquato and G. Stell, Lett. Appl. Eng. Sci. 23, 375 (1985); J. G. Berryman, J. Phys. D 18, 585 (1985), F. Lado and S. Torquato, Phys. Rev. B 33, 3370 ( 1986); S. Torquato and F. Lado, Phys. Rev. B 33, 6248 (1986).

ikB. Lu and S. Torquato, J. Chem. Phys. 93, 3452 (1990).

1"F. Lado and S. Torquato, J. Chem. Phys. 93, 5912 (1990).

${ }^{18}$ J. L. Lebowitz and J. W. Perram, Mol. Phys. 50, 1207 (1983).

${ }^{19}$ See, e.g., J. P. Hansen and I. R. McDonald, Theory of Simple Liquids (Acadmic, London, 1986).

${ }^{20}$ S. Torquato, J. Chem. Phys. 81, 5079 (1984); 84, 6345 (1986)

${ }^{21}$ S. Torquato and G. Stell, J. Chem. Phys. 79, 1505 (1983).

${ }^{22}$ L. Verlet and J. J. Weis, Phys. Rev. A 5, 939 (1972).

${ }^{23}$ See, for example, J. G. Berryman, Phys. Rev. A 27, 1053 (1983), and references therein.

${ }^{24}$ S. Torquato, Phys. Rev. Lett. 64, 2644 (1990).

${ }^{25}$ B. U. Felderhof and J. M. Deutch, J. Chem. Phys. 64, 4551 (1976); D. F. Calef and J. M. Deutch, Annu. Rev. Phys. Chem. 34, 493 (1983); G. H. Weiss, J. Stat. Phys. 42, 3 (1986).

${ }^{26}$ G. H. Fredickson and E. S. G. Shaqfeh, Phys. Fluids A 1, 3 (1989).

${ }^{27}$ Although they explicitly stated the void bound for the case of statistically isotropic media, it is clear that their derivation is general enough to treat anisotropic media.

${ }^{28}$ Z. Hashin and S. Shtrikman, J. Appl. Phys. 33, 1514 (1962).

${ }^{29}$ J. R. Willis, J. Mech. Phys. Solids 25, 185 (1977).

${ }^{30}$ J. D. Eshelby, Proc. R. Soc. London Ser. A 241, 376 (1957).

${ }^{31}$ P. Debye, H. R. Andersen, and H. Brumberger, J. Appl. Phys. 28, 679 (1957). 